\title{
SNARE-CNN: a 2D convolutional neural network architecture to identify SNARE proteins from high-throughput sequencing data
}

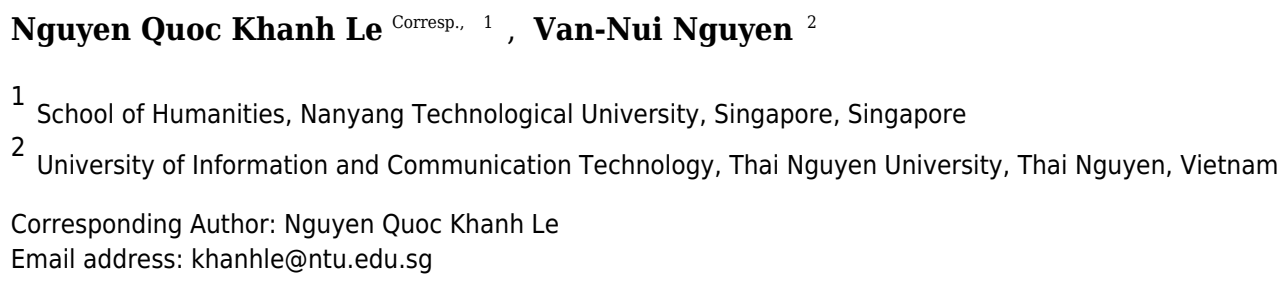

Deep learning has been increasingly and widely used to solve numerous problems in various fields with state-of-the-art performance. It can also be applied in bioinformatics to reduce the requirement for feature extraction and reach high performance. This study attempts to use deep learning to predict SNARE proteins, which is one of the most vital molecular functions in life science. A functional loss of SNARE proteins has been implicated in a variety of human diseases (e.g., neurodegenerative, mental illness, cancer, and so on). Therefore, creating a precise model to identify their functions is a crucial problem for understanding these diseases, and designing the drug targets. Our SNARE-CNN model which uses two-dimensional convolutional neural networks and position-specific scoring matrices profiles could identify SNARE proteins with achieved sensitivity of $76.6 \%$, specificity of $93.5 \%$, accuracy of $89.7 \%$, and MCC of 0.7 in cross-validation dataset. We also evaluate the performance of our model via an independent dataset and the result show that we are able to solve the overfitting problem. Compared with other state-of-theart methods, this approach achieved significant improvement in all of the metrics. Throughout the proposed study, we provide an effective model for identifying SNARE proteins and a basis for further research that can apply deep learning in bioinformatics, especially in protein function prediction. SNARE-CNN are freely available at https://github.com/khanhlee/snare-cnn . 
1 SNARE-CNN: a 2D convolutional neural network

2 architecture to identify SNARE proteins from high-

3 throughput sequencing data

4

5

6

7

8

9

10

11

12

13

14

15

16

17

18

19

20

21

22

23

24

25

26

27

28

29

30

31

32

33

34

35

36

37

Nguyen Quoc Khanh Le' ${ }^{1}$, Van-Nui Nguyen ${ }^{2}$

${ }^{1}$ School of Humanities, Nanyang Technological University, 48 Nanyang Ave, Singapore 639798

${ }^{2}$ University of Information and Communication Technology, Thai Nguyen University, Vietnam

Corresponding Author:

Nguyen Quoc Khanh Le ${ }^{1}$

${ }^{1}$ School of Humanities, Nanyang Technological University, 48 Nanyang Ave, Singapore 639798

Email address: khanhle@ntu.edu.sg

\section{Abstract}

Deep learning has been increasingly and widely used to solve numerous problems in various fields with state-of-the-art performance. It can also be applied in bioinformatics to reduce the requirement for feature extraction and reach high performance. This study attempts to use deep learning to predict SNARE proteins, which is one of the most vital molecular functions in life science. A functional loss of SNARE proteins has been implicated in a variety of human diseases (e.g., neurodegenerative, mental illness, cancer, and so on). Therefore, creating a precise model to identify their functions is a crucial problem for understanding these diseases, and designing the drug targets. Our SNARE-CNN model which uses two-dimensional convolutional neural networks and position-specific scoring matrices profiles could identify SNARE proteins with achieved sensitivity of $76.6 \%$, specificity of $93.5 \%$, accuracy of $89.7 \%$, and MCC of 0.7 in cross-validation dataset. We also evaluate the performance of our model via an independent dataset and the result show that we are able to solve the overfitting problem. Compared with other state-of-the-art methods, this approach achieved significant improvement in all of the metrics. Throughout the proposed study, we provide an effective model for identifying SNARE proteins and a basis for further research that can apply deep learning in bioinformatics, especially in protein function prediction. SNARE-CNN are freely available at

https://github.com/khanhlee/snare-cnn.

\section{Introduction}

Deep learning is an advanced machine learning and artificial intelligent technique to learn the representative data with multiple layers of neural networks (LeCun, Bengio, \& Hinton, 2015). Numerous difficult problems have been solved with deep learning, e.g., speech recognition, visual object recognition, object detection. The advantages of deep learning are: (1) significantly 
38

39

40

41

42

43

44

45

46

47

48

49

50

51

52

53

54

55

56

57

58

59

60

61

62

63

64

65

66

67

68

69

70

71

72

73

74

75

76

77

outperforms other solutions in multiple domains, (2) reduces the requirement for feature extraction and time consumption with the use of graphic processing units (GPUs), and (3) easily adapts to a new problem. Deep neural network models often achieve better performance compared to shallow networks, especially in most of problems with big data. Therefore, deep learning becomes popular and attracts numerous huge companies establishing their directions in this field in recent years. Nowadays, much progress towards deep learning has been made using different deep neural network architectures. A number of studies showed that using deep learning can enhance results in various fields, e.g., prediction of cervical cancer diagnosis (Fernandes, Chicco, Cardoso, \& Fernandes, 2018), piRNA (Wang, Hoeksema, \& Liang, 2018), and isolated guitar transcription (Burlet \& Hindle, 2017). Hence, deep learning is also a fascinating trend in bioinformatics and computational biology research. This study attempts to present a framework to apply deep learning in bioinformatics by using two-dimensional convolutional neural network (2D CNN), which is one popular type of deep neural networks. We anticipate our method will lead to a significant improvement when compared to traditional machine learning techniques in the bioinformatics field.

In earlier years, researchers used shallow neural networks for solving a number of problems in bioinformatics and computational biology. For example, Ou constructed QuickRBF package (Oyang, Hwang, Ou, Chen, \& Chen, 2005) for training radial basis function (RBF) networks and applied them on several bioinformatics problems including classifying electron transport proteins (Le, Nguyen, \& Ou, 2017), transporters (Le, Sandag, \& Ou, 2018), and binding sites (Le \& Ou, 2016a, 2016b). (Chang \& Lin, 2011) introduced LibSVM to help biologists implement bioinformatics models by using support vector machines. Recently, as deep learning has been successfully applied in various fields, researchers started to use it in bioinformatics problems, e.g., prediction of piRNA (Wang et al., 2018) and ab initio protein secondary structure (Spencer, Eickholt, \& Cheng, 2015). Although those studies achieved very good performances, we believe that we can obtain superior results by using 2D CNN in some bioinformatics applications. In this study, we applied our architecture in the prediction of SNARE proteins, which is one of the most vital molecules in the life sciences.

SNARE is an evolutionary superfamily of small proteins that have a conservation pattern of 6070 amino acids (SNAP motifs) in their cytoplasmic domain. SNARE proteins catalyze cell membrane integration in eukaryotes and are essential for a wide range of cellular processes, including cell growth, cytokinesis, and synaptic transmission (Jahn \& Scheller, 2006; Wickner \& Schekman, 2008). Most SNAREs contain only one SNARE motif adjacent to a single C-terminal membrane (e.g., synaptobrevin 2 and syntaxin 1). Some SNAREs contain two SNARE motifs that are connected by a long linkage and non-transmembrane sequence (e.g., SNAP-25) but are attached to the membrane through a post-translational modification such as palmitoylation. Various types of SNARE proteins now identified and several studies demonstrated that a functional loss of SNARE proteins has been implicated in numerous diseases (e.g., neurodegenerative (Hou, Wang, Liu, Wang, \& Long, 2017), mental illness (Honer et al., 2002), cancer (Meng \& Wang, 2015; Sun et al., 2016), and so on). Therefore, SNARE proteins play an 
78 important function in the cell and there is a need to develop some bioinformatics techniques to 79 identify them.

80 Because of the essential role in human diseases, SNARE proteins attracted various researchers 81 who conducted their research on them. For instance, Kloepper team attempted to build a database 82 to store and classify SNARE proteins (Kienle, Kloepper, \& Fasshauer, 2009; Kloepper, Kienle, 83 \& Fasshauer, 2007, 2008). Next, (van Dijk, Bosch, ter Braak, van der Krol, \& van Ham, 2008) 84 built a framework to predict functions of SNAREs in sub-Golgi localization. Moreover, (Weimbs 85 et al., 1997) used bioinformatics techniques to analyze conserved domains in SNARE.

86 (Yoshizawa et al., 2006) extracted sequence motifs and the phylogenetic features of

87 SNARE-dependent membrane trafficking. (Shi, Halder, Yavuz, Jahn, \& Shuman, 2016) directed

88 targeting of membrane fusion by SNARE mimicry by convergent evolution of Legionella 89 effectors. (Lu, 2015) analyzed the destructive effect of botulinum neurotoxins on the SNARE 90 91

92

93

94

95

96

97

98

99

100

101

102

103

104

105

106

107

108

109

110

111

112

113

114

115

116

117 protein and proposed that the truncated SNAP-25 mutants will disrupt the assembly of the SNARE core complex, and then inhibit the synaptic membrane fusion accordingly.

Most published works on SNARE proteins achieved high performance, but to our knowledge, no researcher conducted the prediction of SNARE proteins using machine learning techniques. It is challenging and motivates us to create a precise model for this. Besides that, we also applied deep learning in this problem, which is a modern technique for classification and obtain high accuracies in various fields. Based on the advantages of deep learning, this study consequently proposes the use of a $2 \mathrm{D}$ convolutional neural network (CNN) constructed from position-specific scoring matrix (PSSM) profiles to identify SNARE proteins. The basic principle has already been successfully applied to identify electron transporting proteins (Le, Ho, \& Ou, 2017) and Rab GTPases (Le, Ho, \& Ou, 2018). Thus, in this paper, we extend this approach to identify the molecular functions of SNARE proteins. The main achievements, including contributions to the field, are presented as follows: (i) development of a deep learning framework to identify SNARE functions from protein sequences, in which our model exhibited a significant improvement beyond traditional machine learning algorithms; (ii) first computational study to identify SNARE proteins and provide useful information to biologists to discover the SNARE molecular functions; (iii) valid benchmark dataset to train and test SNARE proteins with high accuracy, which forms a basis for future research on SNARE proteins.

As shown in a series of recent publications (W. Chen, Ding, Zhou, Lin, \& Chou, 2018; Cheng, Xiao, \& Chou, 2018a, 2018b; Chou, Cheng, \& Xiao, 2018; P. Feng et al., 2018; Jia, Li, Qiu, Xiao, \& Chou, 2019; Khan, Rasool, Hussain, Khan, \& Chou, 2018; Xiao, Xu, et al., 2018), to develop a really useful statistical predictor for a biological system, one should observe the guidelines of Chou's 5-step rule (Chou, 2011) to make the following five steps very clear: (i) how to construct or select a valid benchmark dataset to train and test the predictor; (ii) how to formulate the statistical samples with an effective mathematical expression that can truly reflect their intrinsic correlation with the target to be predicted; (iii) how to introduce or develop a powerful algorithm (or engine) to operate the prediction; (iv) how to properly perform crossvalidation tests to objectively evaluate the anticipated accuracy of the predictor; (v) how to 
118 provide source code and dataset that are accessible to the public. Below, we are to describe how

119 to deal with these steps one-by-one.

\section{Materials \& Methods}

121 We implemented an efficient framework for identifying SNARE proteins by using a 2D CNN

122

123

124

125

126

127

128

129

130

131

132

133

134

135

136

137

138

139

140

141

142

143

144

145

146

147

148

149

150

151

152

153

154

155

and PSSM profiles. The framework consists of four procedures: data collection, feature extraction, $\mathrm{CNN}$ generation, and model evaluation. Fig. 1 presents the flowchart of our framework, and its details are described as follows.

\section{Dataset}

The dataset was retrieved from the UniProt database (by 22-10-2018) (Consortium, 2014), which is one of the comprehensive resources for the protein sequence. First of all, we collected all SNAREs proteins from the UniProt annotation (by using keyword "SNARE"). Note that only reviewed proteins (records with information extracted from literature and curator-evaluated computational analysis) were collected. Subsequently, BLAST (Altschul et al., 1997) was applied to remove the redundant sequences with similarity more than $30 \%$, and the rest of the proteins reaches 245 SNAREs. However, after this process, the rest of proteins only reaches 245 SNAREs, and the number of data points was insufficient for a precise deep learning model. Hence, we used a cut-off level of $100 \%$ in the cross-validation dataset for more data to create a significant model. We still used similarity of $30 \%$ in the independent dataset to evaluate the performance of the model. This step is a very important step to check if the model was overfitting or not.

The proposed problem was the binary classification between SNARE proteins and general proteins, thus we collected a set of general proteins as negative data. In order to create a precise model, there is a need to collect negative dataset which has a similar function and structure with the positive dataset. From that, it is challenging to build a precise model but it increases our contribution to the predictor. It will also help us decrease the number of negative data collected. After considering the structure and function, we chose vesicular transport protein, which is a general protein including SNARE protein. We counted it as negative data to perform the classification problem. We removed the redundant data between two datasets as well as the sequences with similarity more than $30 \%$. Finally, there were 682 SNARE proteins and 2583 non-SNARE proteins used. We then divided data into cross-validation and independent dataset. The detail of the dataset using in this study is listed in Table 1.

\section{Encoding feature sets from the protein sequence information}

In order to convert the protein sequence information into feature sets, we applied the PSSM matrices for FASTA sequences. A PSSM profile is a matrix represented by all motifs in biological sequences in general and in protein sequences in particular. It is created by rendering two sequences having similar structures with different amino acid compositions. Therefore, PSSM profiles have been adopted and used in a number of bioinformatics problems, e.g., prediction of protein secondary structure (Jones, 1999), protein disorder (Shimizu, Hirose, \& 
156 Noguchi, 2007), and transport protein (Ou, Chen, \& Gromiha, 2010) with significant

157 improvements.

158 Since the retrieved dataset is in FASTA format, it needs to be converted into PSSM profiles. To 159 perform this task, we used PSI-BLAST (Altschul et al., 1997) to search all the sequence

160 alignments of proteins in the non-redundant (NR) database with two iterations. The query to 161 produce the PSSM profile is as follows:

162 psiblast.exe -num_iterations $2-d b<n r>-i n \_m s a<f a s t a \_f i l e>-o u t$ ascii_ $<p s s m \_f i l e>$

163 The feature extraction part of Fig. 1 indicates the information of generating the 400 PSSM

164 capabilities from original PSSM profiles. Each amino acid in the sequence is represented by a

165 vector of 20 values (each row). First, we summed up all rows with the same amino acid to

166 transform the original PSSM profiles to PSSM profiles with 400 dimensions. The purpose of this

167 step is to force this data type into something easier for the neural network to deal with. Each

168 element of the 400D input vector was then divided by the sequence length and then be scaled

169 before inserting into neural networks.

\section{Input layers for 2D convolutional neural networks}

171 The architecture of our CNN is described in the below part of Fig. 1. The CNN contains three

172 layers: an input layer, hidden layers (including convolutional, pooling and fully connected

173 layers), and an output layer. CNN had been applied in numerous applications in various fields

174 and convinced wonderful results (Amidi et al., 2018; Palatnik de Sousa, 2018). In our study, an

175 input of the CNN is a PSSM corresponding to the protein sequences. We then propose a method

176 to predict SNARE proteins by using their PSSM profiles as the input data. With this type of

177 dataset, we assumed the PSSM profile with 20x20 matrix as a grayscale image with 20x20

178 pixels, we can then train the model with two-dimensional CNN. The input PSSM profile was

179 then connected to our 2D CNN in which we set a variety of parameters to improve the

180 performance of the model. By using a 2D CNN rather than other neural network structures, we

181 aimed to capture as many hidden spatial features as possible in the PSSM matrices. This

182 approach guarantees the correctness of the generated features and prevents the disorder problem

183 inside the amino acid sequences. The more hidden layers generated, the more hidden features

184 generated in CNN to identify SNARE proteins easily. In this work, we used four filter layers

185 (with 32, 64, 128, and 256 filters) and three different kernel sizes in each filter.

\section{Multiple hidden layers for deep neural networks}

187 Following the input layer, hidden layers aim to generate matrices to learn the features. We 188 established the hidden layers that contained various sub-layers with different parameters and 189 shapes. Those 2D sub-layers are zero padding, convolutional, max pooling and fully-connected 190 layers with different numbers of filters. All of the layers are combined together to become the 191 nodes in the deep neural networks. The quality of our model was determined by the number of 192 layers and parameters. The first layer of our 2D CNN architecture is the zero padding 2D layer, 193 which added zero values at the beginning and the end of 20x20 matrices. The shape matrix 194 changed to $22 \times 22$ dimensions when we added the zero padding layer into our network. After we 
195

196

197

198

199

200

201

202

203

204

205

206

207

208

209

210

211

212

213

214

215

216

217

218

219

220

221

222

223

224

225

226

applied the filters into the input shape, the output dimension was not different under the effect of the zero padding.

$$
z p=\frac{k-1}{2}
$$

where $\mathrm{k}$ is the filter size. Next, the 2D convolution layer was used with a kernel size of $3 \times 3$, meaning that the features will be learned with the $3 \times 3$ matrices and sliced to the end. After each step, the next layer will take the weights and biases from its previous layer and train again. Normally, a 2D max-pooling layer follows the 2D convolution layer. There are several parameters for a max-pooling layer, i.e., loop size and stride. In our study, we performed max pooling by a stride of 2 through the selection of the maximum value over a window of 22 . By using this process, we can reduce the processing time in the next layers. The output size of a convolutional layer is computed as follow.

$$
o s=\frac{w-k+2 p}{s}+1
$$

where $\mathrm{w}$ is the input size, $\mathrm{k}$ is the filter size, $\mathrm{p}$ is the padding and $\mathrm{s}$ is the stride size.

\section{Output layers}

The first layer in the output layer is a flatten layer. A flatten layer is always included before fully connected layers to convert the input matrix into a vector. We applied two fully connected layers in which each node is fully-connected to all the nodes of the previous layer. Fully connected layers are typically used in the last stages of CNNs. All the nodes of the first layer are connected to the flatten layer to allow the model to gain more knowledge and perform better. The second layer connects the first fully-connected layer to the output layer. Moreover, we inserted the next layer, dropout, to enhance the performance results of the model and it also helps our model prevent overfitting (Srivastava, Hinton, Krizhevsky, Sutskever, \& Salakhutdinov, 2014). In the dropout layer, the model will randomly deactivate a number of neurons with a certain probability p. By tuning the dropout value (from 0 to 1), we will save a lot of computing time for the next layers, and the training time will be faster. Furthermore, an additional non-linear operation called ReLU (Rectified Linear Unit) was performed after each convolution operation. To define the ReLU output, we used this formula:

$$
f(x)=\max (0, x)
$$

where $\mathrm{x}$ is the number of inputs into a neural network. The output of the model was computed through a softmax function by which the probability for each possible output was determined. The softmax function is a logistic function which is defined by the following formula:

$$
\sigma(z)_{i}=\frac{e^{z_{i}}}{\sum_{k=1}^{K} e^{z_{k}}}
$$


227

228

229

230

231

232

233

234

235

236

237

238

239

240

241

242

243

244

245

246

247

248

249

250

251

252

253

254

255

256

257 where $\mathrm{z}$ is the input vector with $\mathrm{K}$-dimensional vector, $\mathrm{K}$-dimensional vector $\sigma(\mathrm{z})$ is real values in the range $(0,1)$ and $j^{\text {th }}$ class is the predicted probability from sample vector $x$. In summary, we set a total of 233,314 trainable parameters in the model (Table 2).

\section{Performance evaluation}

The most important purpose of the present study was to predict whether or not a sequence is SNARE protein; therefore, we used "Positive" to define the SNARE protein, and "Negative" to define the non-SNARE protein. For each dataset, we first trained the model by applying 5-fold cross-validation technique on the training dataset. Based on the 5-fold crossvalidation results, hyper-parameter optimization process was employed to find the best model for each dataset. Finally, the independent dataset was used to assess the predictive ability of the current model.

Based on the Chou's symbols introduced for studying protein signal peptides (Chou, 2001), a set of four intuitive metrics were derived, as given in Eq.14 of (W. Chen, Feng, Lin, \& Chou, 2013) or in Eq.19 of (Xu, Shao, Wu, Deng, \& Chou, 2013). For evaluating the performance of the methods, we also adopted Chou's criterion used in many bioinformatics studies (J. Chen, Liu, Yang, \& Chou, 2007; P.-M. Feng, Chen, Lin, \& Chou, 2013; Taju, Nguyen, Le, Kusuma, \& Ou, 2018). Either the set of traditional metrics copied from math books or the intuitive metrics derived from the Chou's symbols (Eqs.5-8) are valid only for the single-label systems (where each sample only belongs to one class). For the multi-label systems (where a sample may simultaneously belong to several classes), whose existence has become more frequent in system biology (Cheng, Xiao, \& Chou, 2017a, 2017b; Cheng, Zhao, Lin, Xiao, \& Chou, 2017; Xiao, Cheng, Chen, Mao, \& Chou, 2018), system medicine (Cheng, Zhao, Xiao, \& Chou, 2017) and biomedicine (Qiu, Sun, Xiao, Xu, \& Chou, 2016), a completely different set of metrics as defined in (Chou, 2013) is absolutely needed. Some standard metrics were used, such as sensitivity, specificity, accuracy, and Matthews correlation coefficient (MCC) using below given formulae (TP, FP, TN, FN are true positive, false positive, true negative, and false negative values, respectively):

Sensitivity $=1-\frac{N_{-}^{+}}{N^{+}}, 0 \leq \operatorname{Sen} \leq 1$

$$
1-\left(\frac{N_{-}^{+}}{N^{+}}+\frac{N_{+}^{-}}{N^{-}}\right)
$$

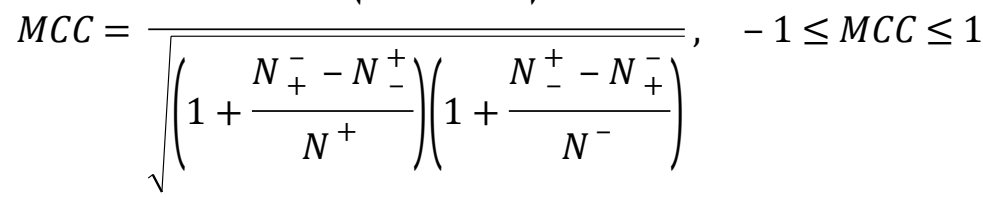


258 The relations between these symbols and the symbols in Eqs. (5, 6, 7 and 8) are given by:

259

$$
\left\{\begin{array}{c}
N_{+}^{-}=F P \\
N_{-}^{+}=F N \\
N^{+}=T P+N_{-}^{+} \\
N^{-}=T N+N_{+}^{-}
\end{array}\right.
$$

\section{Results and Discussions}

261

262

263

264

265

266

267

268

269

270

271

272

273

274

275

276

277

278

279

280

281

282

283

284

285

286

287

288

289

The quality and reliability of the modeling techniques of research is an important factor in the study. Initially, we designed an experiment by analyzing data, perform calculations and take various comparisons in the results and discussions section.

\section{Composition of amino acid in SNARE and non-SNARE proteins}

We analyzed the composition of amino acid and the variance of amino acid composition in SNARE sequences and non-SNARE sequences by computing the frequency between them. Fig. 2 illustrates the amino acids which contributed the significantly highest frequency in two different datasets. We realized that the amino acid $\mathrm{E}$, and $\mathrm{K}$, and $\mathrm{P}$ occur at the highest frequencies surrounding the SNARE proteins. On the other hand, amino acids $\mathrm{G}$ and $\mathrm{P}$ occur at the highest frequencies surrounding the non-SNARE proteins. Therefore, these amino acids certainly had an essential role in identifying SNARE proteins. Thus, our model might predict SNARE proteins accurately via the special features from those amino acids contributions.

\section{Performance for identifying SNARE proteins with 2D CNN}

We implemented our 2D CNN architecture by using Keras package with Tensorflow backend. First, we tried to find the optimal setup for the hidden layers by doing experiments using four different filter sizes: $32,64,128$, and 256 . Table 3 demonstrates the performance results from various filter layers in the cross-validation dataset. We easily observe that during the 5 -fold cross-validation to identify SNAREs, the model with 256 filters was prominent identifying these sequences with an average 5 -fold cross-validation accuracy of $88.2 \%$. The performance results are higher than the performances from the other results with other filters. The sensitivity, specificity, and MCC for cross-validation data achieved 70.5\%, 93.3\%, and 0.65, respectively. Therefore, we used 256 filters for the hidden layer to develop our model. We then optimized the neural networks using a variety of optimizers: rmsprop, adam, nadam, sgd, and adadelta. The model was reinitialized, i.e. a new network is built, after each round of optimization so as to provide a fair comparison between the different optimizers. Overall, the performance results are shown in Fig. 3 and we decided to choose nadam, an optimizer with consistent performance to create our final model.

Improving the performance results and preventing over-fitting problem with dropout 
290 It can be seen that there was a fair difference in performance between using the cross-validation 291 dataset and the independent dataset. It is due to the non-removing similarity in cross-validation, 292 and now we address this issue. To solve this issue, we applied an important technique called 293 dropout (Srivastava et al., 2014). Table 4 presents the performances of the model when we varied

294

295

296

297

298

299

300

301

302

303

304

305

306

307

308

309

310

311

312

313

314

315

316

317

318

319

320

321

322

323

324

325

326

327

the dropout value from 0 to 1 . It can be seen that the performance from the dropout value of 0.1 was higher than others, with the sensitivity, specificity, accuracy, and MCC of $72.4 \%, 94.4 \%$, $89.5 \%$, and 0.69 , respectively. In the independent dataset, the sensitivity, specificity, accuracy, and MCC were $44.7 \%, 95.4 \%, 90.4 \%$, and 0.43 , respectively. We can see that the performance of the independent dataset has been already improved and moved closer to that of the crossvalidation dataset. Therefore, the over-fitting problem was gradually resolved, and we used this dropout value for our final model.

Moreover, the number of epochs used in the experiment extremely affects the performance results. To discover the optimal epoch, we ran our experiments by ranging the epoch value from the first epoch to the $500^{\text {th }}$ epoch. During this process, we saved the checkpoint with the highest performance and used its parameters to create our model. Until this final step, the independent sensitivity, specificity, accuracy, and MCC reached $65.8 \%, 90.3 \%, 87.9 \%$ and 0.46 , respectively. This result is close to that of the cross-validation dataset at the same level of 2D CNN architecture. Finally, our model applied 256 filter layers, nadam optimizer, and dropout value of 0.1 to identify SNARE proteins with the highest performance.

\section{Comparative performance for identifying SNAREs between 2D CNN and shallow neural networks}

We examined the performances of using different machine learning classifiers for identifying SNARE proteins. We used four different classifiers (i.e., nearest neighbor $(\mathrm{kNN})$, Gaussian, Random Forest, and support vector machine (SVM)) to evaluate the model and compared 2D $\mathrm{CNN}$ results with their results. For a fair comparison, we definitely used the optimal parameters for all the classifiers in all the experiments. Table 5 shows the performance results between our method and other machine learning algorithms. It can be seen that our 2D CNN exhibited higher performance than those of the other traditional machine learning techniques using the same experimental setup. Especially, our 2D CNN outperformed other algorithms when using the independent dataset.

\section{Comparative performance for identifying SNAREs between 2D CNN and BLAST search pipeline}

To make our prediction have convincing, we aim to simply BLASTing the SNARE and nonSNARE sequences. The objective of this step is to check whether the first non-identical match was a SNARE/non-SNARE protein. We then compared with our PSSM via PSI-BLAST and the performance results were shown in Table 6. It is easy to say that we are able to reach a better performance when using the PSSM profiles to build a classifier. It also means that BLAST can search a sequence within motifs, but it cannot capture hidden information in sequences. 
328 Therefore, it is necessary and useful to create an advanced classifier with stronger features e.g., 329 PSSM profiles in this study.

330 Furthermore, source codes and publicly accessible web-servers represent the current trend for 331 developing various computational methods (W. Chen et al., 2018; Cheng et al., 2018a, 2018b; 332 Chou et al., 2018; P. Feng et al., 2018; Jia et al., 2019; Khan et al., 2018; Le, Ho, \& Ou; Xiao, $333 \mathrm{Xu}$, et al., 2018). Actually, they have significantly enhanced the impacts of computational 334 biology on medical science (Chou, 2015), driving medicinal chemistry into an unprecedented 335 revolution (Chou, 2017), here we also publish our source codes and dataset at

336 https://github.com/khanhlee/snare-cnn for presenting the new method reported in this paper.

\section{Conclusions}

338 Deep learning, a leading technique in various fields, has been increasingly applied in

339 bioinformatics and computational biology. This study approaches a novel for identifying SNARE

340

341

342

343

344

345

346

347

348

349

350

351

352

353

354

355

356

357

358

359

360

361

362

363

364

365

366

367

proteins by using deep learning. The idea is to transform PSSM profiles into matrices and use them as the input to 2D CNN architectures. We evaluated the performance of our model, which was developed by using a 2D CNN and PSSM profiles, using 5-fold cross-validation and an independent testing dataset. Our method produced superior performance, and compared to other state-of-the-art neural networks, it achieved a significant improvement in all the typical measurement metrics. Using our model, new SNARE proteins can be accurately identified and used for drug development. Moreover, the contribution of this study could help further research to promote the use of 2D CNN in bioinformatics, especially in protein function prediction.

\section{Acknowledgements}

Not applicable.

\section{References}

Altschul, S. F., Madden, T. L., Schäffer, A. A., Zhang, J., Zhang, Z., Miller, W., \& Lipman, D. J. (1997). Gapped BLAST and PSI-BLAST: a new generation of protein database search programs. Nucleic acids research, 25(17), 3389-3402.

Amidi, A., Amidi, S., Vlachakis, D., Megalooikonomou, V., Paragios, N., \& Zacharaki, E. I. (2018). EnzyNet: enzyme classification using 3D convolutional neural networks on spatial representation. PeerJ, 6, e4750. doi:10.7717/peerj.4750

Burlet, G., \& Hindle, A. (2017). Isolated guitar transcription using a deep belief network. PeerJ Computer Science, 3, e109. doi:10.7717/peerj-cs.109

Chang, C.-C., \& Lin, C.-J. (2011). LIBSVM: a library for support vector machines. ACM transactions on intelligent systems and technology (TIST), 2(3), 27.

Chen, J., Liu, H., Yang, J., \& Chou, K.-C. (2007). Prediction of linear B-cell epitopes using amino acid pair antigenicity scale. Amino acids, 33(3), 423-428.

Chen, W., Ding, H., Zhou, X., Lin, H., \& Chou, K.-C. (2018). iRNA(m6A)-PseDNC: Identifying N6-methyladenosine sites using pseudo dinucleotide composition. Analytical

Biochemistry, 561-562, 59-65. doi:https://doi.org/10.1016/j.ab.2018.09.002 
368

369

370

371

372

373

374

375

376

377

378

379

380

381

382

383

384

385

386

387

388

389

390

391

392

393

394

395

396

397

398

399

400

401

402

403

404

405

406

407

408

409

410

411

412

413

414

415

416

417

Chen, W., Feng, P.-M., Lin, H., \& Chou, K.-C. (2013). iRSpot-PseDNC: identify recombination spots with pseudo dinucleotide composition. Nucleic acids research, 41(6), e68-e68. doi:10.1093/nar/gks 1450

Cheng, X., Xiao, X., \& Chou, K.-C. (2017a). pLoc-mPlant: predict subcellular localization of multi-location plant proteins by incorporating the optimal GO information into general PseAAC. Molecular BioSystems, 13(9), 1722-1727. doi:10.1039/C7MB00267J

Cheng, X., Xiao, X., \& Chou, K.-C. (2017b). pLoc-mVirus: Predict subcellular localization of multi-location virus proteins via incorporating the optimal $\mathrm{GO}$ information into general PseAAC. Gene, 628, 315-321. doi:https://doi.org/10.1016/j.gene.2017.07.036

Cheng, X., Xiao, X., \& Chou, K.-C. (2018a). pLoc-mEuk: Predict subcellular localization of multilabel eukaryotic proteins by extracting the key GO information into general PseAAC. Genomics, 110(1), 50-58. doi:https://doi.org/10.1016/j.ygeno.2017.08.005

Cheng, X., Xiao, X., \& Chou, K.-C. (2018b). pLoc-mGneg: Predict subcellular localization of Gram-negative bacterial proteins by deep gene ontology learning via general PseAAC. Genomics, 110(4), 231-239. doi:https://doi.org/10.1016/j.ygeno.2017.10.002

Cheng, X., Zhao, S.-G., Lin, W.-Z., Xiao, X., \& Chou, K.-C. (2017). pLoc-mAnimal: predict subcellular localization of animal proteins with both single and multiple sites. Bioinformatics, 33(22), 3524-3531. doi:10.1093/bioinformatics/btx476

Cheng, X., Zhao, S.-G., Xiao, X., \& Chou, K.-C. (2017). iATC-mISF: a multi-label classifier for predicting the classes of anatomical therapeutic chemicals. Bioinformatics, 33(3), 341346. doi:10.1093/bioinformatics/btw644

Chou, K.-C. (2001). Prediction of signal peptides using scaled window. Peptides, 22(12), 19731979. doi:https://doi.org/10.1016/S0196-9781(01)00540-X

Chou, K.-C. (2011). Some remarks on protein attribute prediction and pseudo amino acid composition. Journal of Theoretical Biology, 273(1), 236-247. doi:https://doi.org/10.1016/j.jtbi.2010.12.024

Chou, K.-C. (2013). Some remarks on predicting multi-label attributes in molecular biosystems. Molecular BioSystems, 9(6), 1092-1100. doi:10.1039/C3MB25555G

Chou, K.-C. (2015). Impacts of Bioinformatics to Medicinal Chemistry. Medicinal Chemistry, 11(3), 218-234. doi:http://dx.doi.org/10.2174/1573406411666141229162834

Chou, K.-C. (2017). An Unprecedented Revolution in Medicinal Chemistry Driven by the Progress of Biological Science. Current Topics in Medicinal Chemistry, 17(21), 23372358. doi:http://dx.doi.org/10.2174/1568026617666170414145508

Chou, K.-C., Cheng, X., \& Xiao, X. (2018). pLoc_bal-mHum: Predict subcellular localization of human proteins by PseAAC and quasi-balancing training dataset. Genomics. doi:https://doi.org/10.1016/j.ygeno.2018.08.007

Consortium, U. (2014). UniProt: a hub for protein information. Nucleic acids research, 43(D1), D204-D212.

Feng, P.-M., Chen, W., Lin, H., \& Chou, K.-C. (2013). iHSP-PseRAAAC: Identifying the heat shock protein families using pseudo reduced amino acid alphabet composition. Analytical Biochemistry, 442(1), 118-125.

Feng, P., Yang, H., Ding, H., Lin, H., Chen, W., \& Chou, K.-C. (2018). iDNA6mA-PseKNC: Identifying DNA N6-methyladenosine sites by incorporating nucleotide physicochemical properties into PseKNC. Genomics. doi:https://doi.org/10.1016/j.ygeno.2018.01.005

Fernandes, K., Chicco, D., Cardoso, J. S., \& Fernandes, J. (2018). Supervised deep learning embeddings for the prediction of cervical cancer diagnosis. PeerJ Computer Science, 4, e154. doi:10.7717/peerj-cs.154

Honer, W. G., Falkai, P., Bayer, T. A., Xie, J., Hu, L., Li, H.-Y., . . Trimble, W. S. (2002). Abnormalities of SNARE Mechanism Proteins in Anterior Frontal Cortex in Severe Mental Illness. Cerebral Cortex, 12(4), 349-356. doi:10.1093/cercor/12.4.349 
418

419

420

421

422

423

424

425

426

427

428

429

430

431

432

433

434

435

436

437

438

439

440

441

442

443

444

445

446

447

448

449

450

451

452

453

454

455

456

457

458

459

460

461

462

463

464

465

466

467

Hou, C., Wang, Y., Liu, J., Wang, C., \& Long, J. (2017). Neurodegenerative Disease Related Proteins Have Negative Effects on SNARE-Mediated Membrane Fusion in Pathological Confirmation. Frontiers in molecular neuroscience, 10, 66.

Jahn, R., \& Scheller, R. H. (2006). SNAREs - engines for membrane fusion. Nature Reviews Molecular Cell Biology, 7, 631. doi:10.1038/nrm2002

Jia, J., Li, X., Qiu, W., Xiao, X., \& Chou, K.-C. (2019). iPPI-PseAAC(CGR): Identify proteinprotein interactions by incorporating chaos game representation into PseAAC. Journal of Theoretical Biology, 460, 195-203. doi:https://doi.org/10.1016/j.jtbi.2018.10.021

Jones, D. T. (1999). Protein secondary structure prediction based on position-specific scoring matrices1. Journal of molecular biology, 292(2), 195-202.

Khan, Y. D., Rasool, N., Hussain, W., Khan, S. A., \& Chou, K.-C. (2018). iPhosT-PseAAC: Identify phosphothreonine sites by incorporating sequence statistical moments into PseAAC. Analytical Biochemistry, 550, 109-116. doi:https://doi.org/10.1016/j.ab.2018.04.021

Kienle, N., Kloepper, T. H., \& Fasshauer, D. (2009). Phylogeny of the SNARE vesicle fusion machinery yields insights into the conservation of the secretory pathway in fungi. BMC evolutionary biology, 9(1), 19.

Kloepper, T. H., Kienle, C. N., \& Fasshauer, D. (2007). An elaborate classification of SNARE proteins sheds light on the conservation of the eukaryotic endomembrane system. Molecular biology of the cell, 18(9), 3463-3471.

Kloepper, T. H., Kienle, C. N., \& Fasshauer, D. (2008). SNAREing the basis of multicellularity: consequences of protein family expansion during evolution. Molecular biology and evolution, 25(9), 2055-2068.

Le, N. Q. K., Ho, Q.-T., \& Ou, Y.-Y. Using two-dimensional convolutional neural networks for identifying GTP binding sites in Rab proteins. Journal of Bioinformatics and Computational Biology, 0. doi:10.1142/s0219720019500057

Le, N. Q. K., Ho, Q.-T., \& Ou, Y.-Y. (2017). Incorporating deep learning with convolutional neural networks and position specific scoring matrices for identifying electron transport proteins. Journal of Computational Chemistry, 38(23), 2000-2006. doi:doi:10.1002/jcc.24842

Le, N. Q. K., Ho, Q.-T., \& Ou, Y.-Y. (2018). Classifying the molecular functions of Rab GTPases in membrane trafficking using deep convolutional neural networks. Analytical Biochemistry, 555, 33-41. doi:https://doi.org/10.1016/j.ab.2018.06.011

Le, N. Q. K., Nguyen, T.-T.-D., \& Ou, Y.-Y. (2017). Identifying the molecular functions of electron transport proteins using radial basis function networks and biochemical properties. Journal of Molecular Graphics and Modelling, 73, 166-178. doi:https://doi.org/10.1016/j.jmgm.2017.01.003

Le, N. Q. K., \& Ou, Y.-Y. (2016a). Incorporating efficient radial basis function networks and significant amino acid pairs for predicting GTP binding sites in transport proteins. BMC Bioinformatics, 17(19), 501. doi:10.1186/s12859-016-1369-y

Le, N. Q. K., \& Ou, Y.-Y. (2016b). Prediction of FAD binding sites in electron transport proteins according to efficient radial basis function networks and significant amino acid pairs. BMC Bioinformatics, 17(1), 298. doi:10.1186/s12859-016-1163-X

Le, N. Q. K., Sandag, G. A., \& Ou, Y.-Y. (2018). Incorporating post translational modification information for enhancing the predictive performance of membrane transport proteins. Computational Biology and Chemistry, 77, 251-260. doi:https://doi.org/10.1016/j.compbiolchem.2018.10.010

LeCun, Y., Bengio, Y., \& Hinton, G. (2015). Deep learning. nature, 521(7553), 436.

Lu, B. (2015). The destructive effect of botulinum neurotoxins on the SNARE protein: SNAP-25 and synaptic membrane fusion. PeerJ, 3, e1065. doi:10.7717/peerj.1065 
468

469

470

471

472

473

474

475

476

477

478

479

480

481

482

483

484

485

486

487

488

489

490

491

492

493

494

495

496

497

498

499

500

501

502

503

504

505

506

507

508

509

510

511

512

513

514

515

516

Meng, J., \& Wang, J. (2015). Role of SNARE proteins in tumourigenesis and their potential as targets for novel anti-cancer therapeutics. Biochimica et Biophysica Acta (BBA) Reviews on Cancer, 1856(1), 1-12. doi:https://doi.org/10.1016/j.bbcan.2015.04.002

Ou, Y. Y., Chen, S. A., \& Gromiha, M. M. (2010). Classification of transporters using efficient radial basis function networks with position-specific scoring matrices and biochemical properties. Proteins: Structure, Function, and Bioinformatics, 78(7), 1789-1797.

Oyang, Y.-J., Hwang, S.-C., Ou, Y.-Y., Chen, C.-Y., \& Chen, Z.-W. (2005). Data classification with radial basis function networks based on a novel kernel density estimation algorithm. IEEE transactions on neural networks, 16(1), 225-236.

Palatnik de Sousa, I. (2018). Convolutional ensembles for Arabic Handwritten Character and Digit Recognition. PeerJ Computer Science, 4, e167. doi:10.7717/peerj-cs.167

Qiu, W.-R., Sun, B.-Q., Xiao, X., Xu, Z.-C., \& Chou, K.-C. (2016). iPTM-mLys: identifying multiple lysine PTM sites and their different types. Bioinformatics, 32(20), 3116-3123. doi:10.1093/bioinformatics/btw380

Shi, X., Halder, P., Yavuz, H., Jahn, R., \& Shuman, H. A. (2016). Direct targeting of membrane fusion by SNARE mimicry: Convergent evolution of Legionella effectors. Proceedings of the National Academy of Sciences, 113(31), 8807.

Shimizu, K., Hirose, S., \& Noguchi, T. (2007). POODLE-S: web application for predicting protein disorder by using physicochemical features and reduced amino acid set of a positionspecific scoring matrix. Bioinformatics, 23(17), 2337-2338.

Spencer, M., Eickholt, J., \& Cheng, J. (2015). A deep learning network approach to ab initio protein secondary structure prediction. IEEE/ACM transactions on computational biology and bioinformatics (TCBB), 12(1), 103-112.

Srivastava, N., Hinton, G., Krizhevsky, A., Sutskever, I., \& Salakhutdinov, R. (2014). Dropout: a simple way to prevent neural networks from overfitting. The Journal of Machine Learning Research, 15(1), 1929-1958.

Sun, Q., Huang, X., Zhang, Q., Qu, J., Shen, Y., Wang, X., . . Ren, B. (2016). SNAP23 promotes the malignant process of ovarian cancer. Journal of Ovarian Research, 9(1), 80. doi:10.1186/s13048-016-0289-9

Taju, S. W., Nguyen, T.-T.-D., Le, N.-Q.-K., Kusuma, R. M. I., \& Ou, Y.-Y. (2018). DeepEfflux: a 2D convolutional neural network model for identifying families of efflux proteins in transporters. Bioinformatics, 34(18), 3111-3117. doi:10.1093/bioinformatics/bty302

van Dijk, A. D. J., Bosch, D., ter Braak, C. J. F., van der Krol, A. R., \& van Ham, R. C. H. J. (2008). Predicting sub-Golgi localization of type II membrane proteins. Bioinformatics, 24(16), 1779-1786. doi:10.1093/bioinformatics/btn309

Wang, K., Hoeksema, J., \& Liang, C. (2018). piRNN: deep learning algorithm for piRNA prediction. PeerJ, 6, e5429. doi:10.7717/peerj.5429

Weimbs, T., Low, S. H., Chapin, S. J., Mostov, K. E., Bucher, P., \& Hofmann, K. (1997). A conserved domain is present in different families of vesicular fusion proteins: a new superfamily. Proceedings of the National Academy of Sciences, 94(7), 3046-3051.

Wickner, W., \& Schekman, R. (2008). Membrane fusion. Nature structural \& molecular biology, $15(7), 658$.

Xiao, X., Cheng, X., Chen, G., Mao, Q., \& Chou, K.-C. (2018). pLoc_bal-mGpos: Predict subcellular localization of Gram-positive bacterial proteins by quasi-balancing training dataset and PseAAC. Genomics. doi:https://doi.org/10.1016/j.ygeno.2018.05.017

Xiao, X., Xu, Z.-C., Qiu, W.-R., Wang, P., Ge, H.-T., \& Chou, K.-C. (2018). iPSW(2L)-PseKNC: A two-layer predictor for identifying promoters and their strength by hybrid features via pseudo K-tuple nucleotide composition. Genomics. doi:https://doi.org/10.1016/j.ygeno.2018.12.001 
517 Xu, Y., Shao, X.-J., Wu, L.-Y., Deng, N.-Y., \& Chou, K.-C. (2013). iSNO-AAPair: incorporating 518 amino acid pairwise coupling into PseAAC for predicting cysteine S-nitrosylation sites in proteins. PeerJ, 1, e171. doi:10.7717/peerj.171

520

521

522

523

Yoshizawa, A. C., Kawashima, S., Okuda, S., Fujita, M., Itoh, M., Moriya, Y., . . Kanehisa, M. (2006). Extracting Sequence Motifs and the Phylogenetic Features of SNAREDependent Membrane Traffic. Traffic, 7(8), 1104-1118. doi:doi:10.1111/j.16000854.2006.00451.x 


\section{List of Figures}

526 Figure 1. Flowchart for identifying SNARE proteins using two-dimensional convolutional

527 neural networks

528 Figure 2. Amino acid composition in SNARE and non-SNARE proteins

529 Figure 3. The validation accuracy on identifying SNARE proteins using different optimizers

530

\section{List of Tables}

532 Table 1. Statistics of all retrieved SNARE and non-SNARE proteins

533 Table 2. All layers and trainable parameters of the two-dimensional convolutional neural

534 networks in this study

535 Table 3. Performance results of identifying SNAREs with different filter layers

536 Table 4. Performance results of identifying SNAREs with different dropout levels

537 Table 5. Comparative performance between 2D CNN and other shallow neural networks

538 Table 6. Comparative performance between our classification method and BLAST search 539 pipeline 


\section{Figure 1}

Flowchart for identifying SNARE proteins using two-dimensional convolutional neural networks

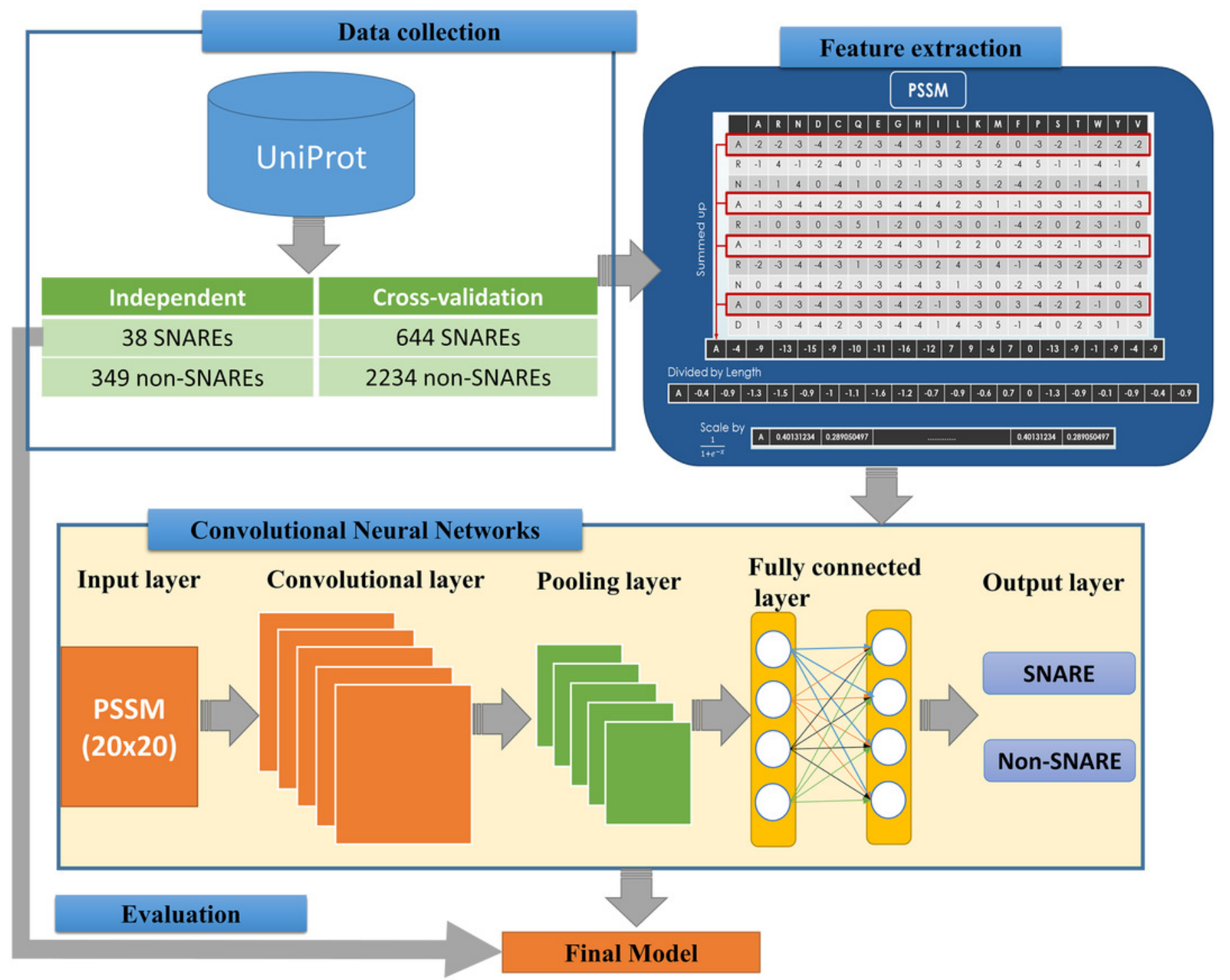


Figure 2

Amino acid composition in SNARE and non-SNARE proteins

12

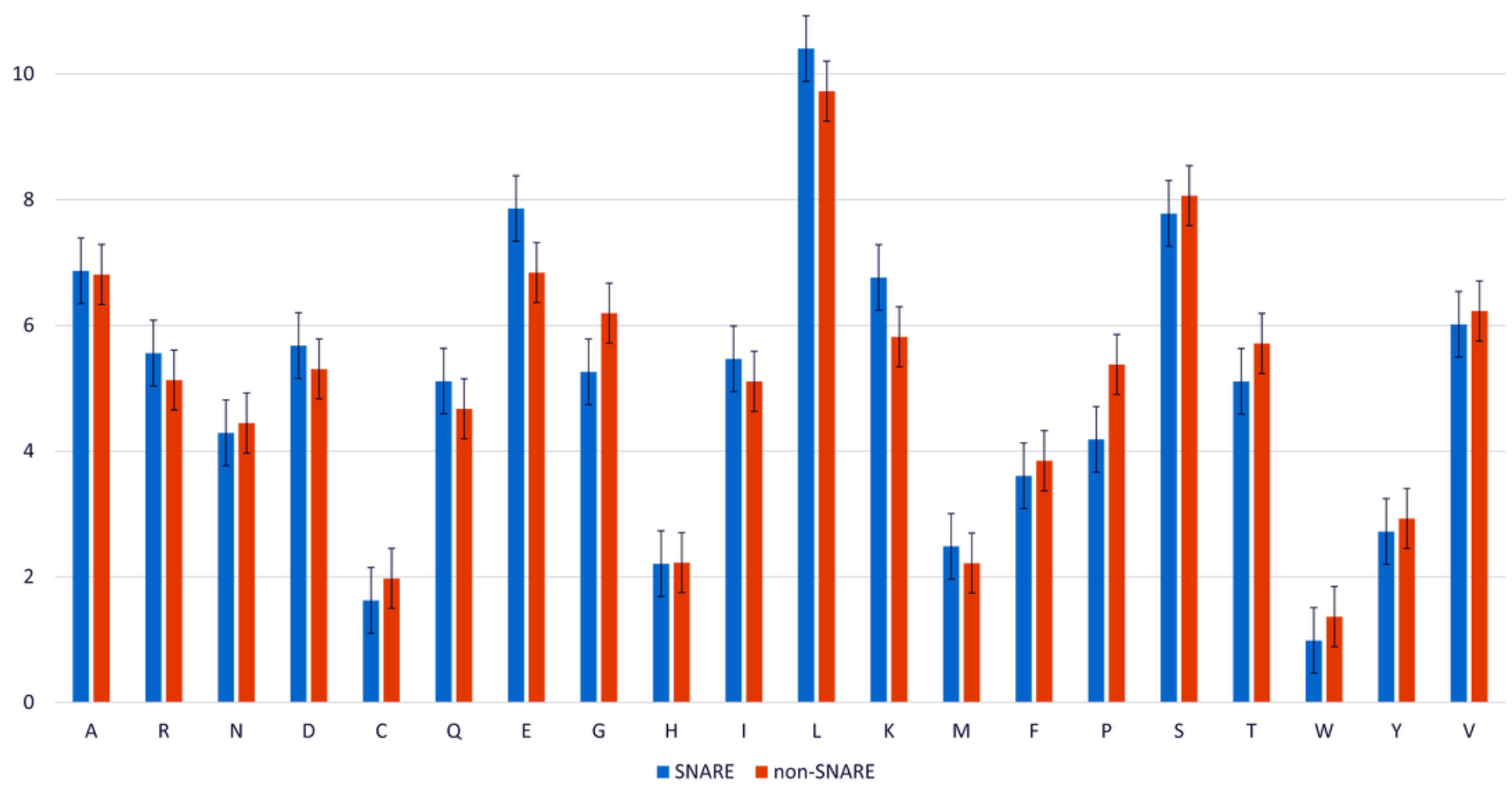


Figure 3

The validation accuracy on identifying SNARE proteins using different optimizers

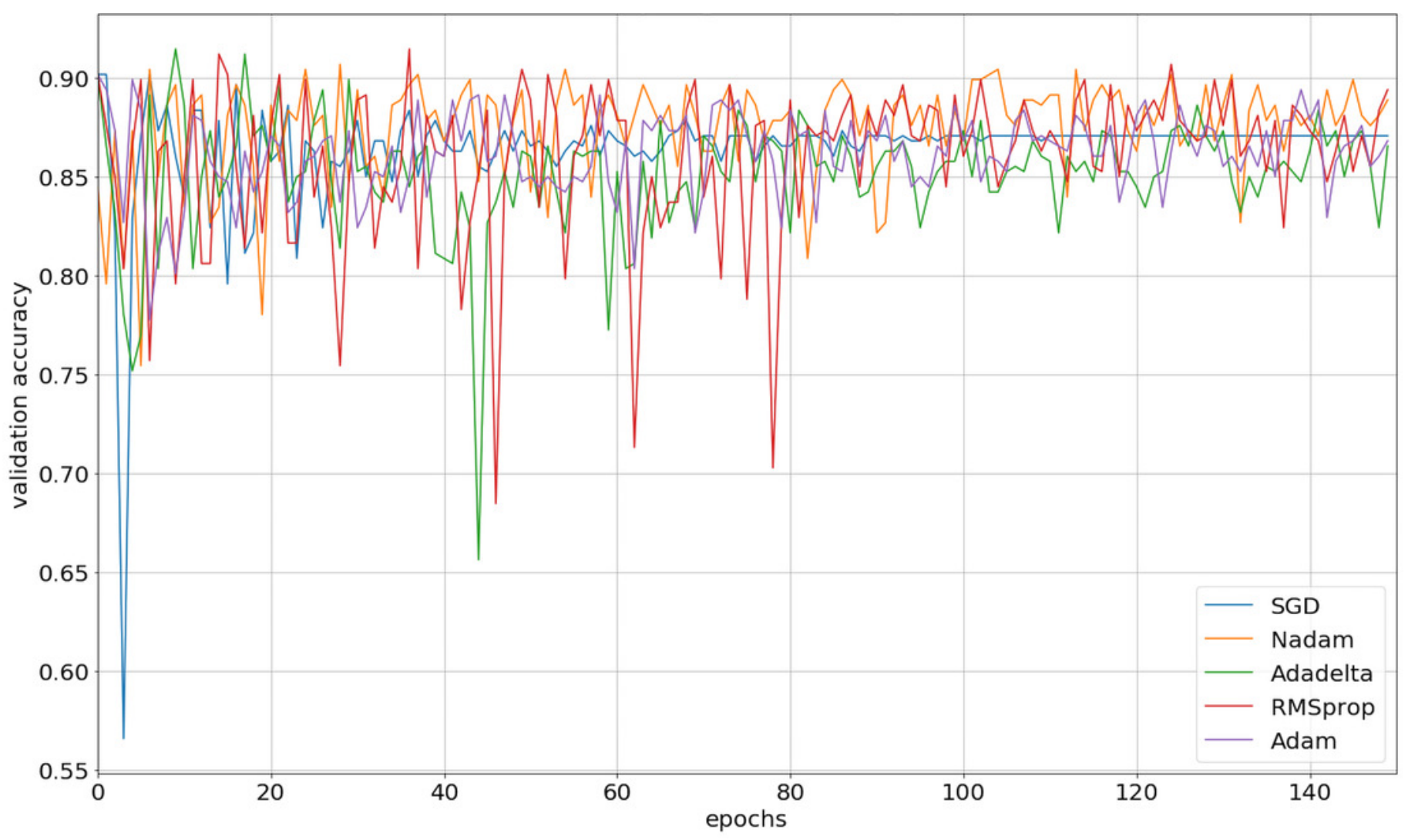




\section{Table $\mathbf{1}$ (on next page)}

Statistics of all retrieved SNARE and non-SNARE proteins 
1 Table 1. Statistics of all retrieved SNARE and non-SNARE proteins

\begin{tabular}{|l|l|l|}
\hline & Cross-validation & Independent \\
\hline SNARE & 644 & 38 \\
\hline Non-SNARE & 2234 & 349 \\
\hline
\end{tabular}

2 


\section{Table 2 (on next page)}

All layers and trainable parameters of the two-dimensional convolutional neural networks in this study 
1 Table 2. All layers and trainable parameters of the two-dimensional convolutional neural 2 networks in this study

\begin{tabular}{|l|l|l|}
\hline Layer (type) & Output shape & Parameters \# \\
\hline Zeropadding2d_1 & (None, 3, 22, 20) & 0 \\
\hline Conv2d_1 & (None, $1,20,32)$ & 5792 \\
\hline Max_pooling2d_1 & (None, $1,10,16)$ & 0 \\
\hline Zeropadding2d_2 & (None, 3, 12, 16) & 0 \\
\hline Conv2d_2 & (None, $1,10,64)$ & 9280 \\
\hline Max_pooling2d_2 & (None, $1,5,32)$ & 0 \\
\hline Zeropadding2d_3 & (None, 3, 7, 32) & 0 \\
\hline Conv2d_3 & (None, 1, 5, 128) & 36992 \\
\hline Max_pooling2d_3 & (None, 1, 2, 64) & 0 \\
\hline Zeropadding2d_4 & (None, 3, 4, 64) & 0 \\
\hline Conv2d_4 & (None, 1, 2, 256) & 147712 \\
\hline Max_pooling2d_4 & (None, 1, 1, 128) & 0 \\
\hline Flatten_1 & (None, 128) & 0 \\
\hline Dense_1 & (None, 256) & 33024 \\
\hline Dropout_1 & (None, 256) & 0 \\
\hline Dense_2 & (None, 2) & 514 \\
\hline Activation_1 & (None, 2) & 0 \\
\hline
\end{tabular}




\section{Table 3 (on next page)}

Performance results of identifying SNAREs with different filter layers 
1 Table 3. Performance results of identifying SNAREs with different filter layers

\begin{tabular}{|l|l|l|l|l|}
\hline Filters & Sens & Spec & Acc & MCC \\
\hline 32 & 68.3 & 91.9 & 86.6 & 0.61 \\
\hline $32-64$ & 69.9 & 93.2 & 88 & 0.65 \\
\hline $32-64-128$ & 73.3 & 91.5 & 87.5 & 0.64 \\
\hline $32-64-128-256$ & 70.5 & 93.3 & 88.2 & 0.65 \\
\hline
\end{tabular}

2 


\section{Table 4 (on next page)}

Performance results of identifying SNAREs with different dropout levels 
1 Table 4. Performance results of identifying SNAREs with different dropout levels

\begin{tabular}{|l|l|l|l|l|l|l|l|l|}
\hline \multicolumn{4}{|l|}{ Cross-validation } & \multicolumn{3}{l|}{ Independent } \\
\hline Dropout & Sens & Spec & Acc & MCC & Sens & Spec & Acc & MCC \\
\hline 0 & 70.5 & 93.3 & 88.2 & 0.65 & 57.9 & 85.7 & 82.9 & 0.33 \\
\hline 0.1 & 72.4 & 94.4 & 89.5 & 0.69 & 44.7 & 95.4 & 90.4 & 0.43 \\
\hline 0.2 & 69.3 & 93.9 & 88.4 & 0.65 & 50 & 87.4 & 83.7 & 0.3 \\
\hline 0.3 & 69.6 & 94.2 & 88.7 & 0.66 & 42.1 & 86 & 81.7 & 0.22 \\
\hline 0.4 & 72 & 92.6 & 88 & 0.65 & 39.5 & 91.4 & 86.3 & 0.29 \\
\hline 0.5 & 69.7 & 94.8 & 89.1 & 0.68 & 36.8 & 92.8 & 87.3 & 0.29 \\
\hline
\end{tabular}

2 


\section{Table 5 (on next page)}

Comparative performance between 2D CNN and other shallow neural networks 
1 Table 5. Comparative performance between $2 \mathrm{D} C \mathrm{CNN}$ and other shallow neural networks

\begin{tabular}{|l|l|l|l|l|l|l|l|l|}
\hline Classifier & Sens & Spec & Acc & MCC & Sens & Spec & Acc & MCC \\
\hline kNN & 60.1 & 95.4 & 87.5 & 0.62 & 28.9 & 95.1 & 88.6 & 0.28 \\
\hline RandomForest & 59.6 & 98.2 & 89.5 & 0.68 & 15.8 & 98 & 89.9 & 0.23 \\
\hline Gaussian & 93.5 & 30.5 & 44.6 & 0.23 & 81.6 & 23.2 & 28.9 & 0.03 \\
\hline SVM & 35.2 & 98.1 & 84 & 0.48 & 28.9 & 97.1 & 90.4 & 0.34 \\
\hline 2D CNN & 76.6 & 93.5 & 89.7 & 0.7 & 65.8 & 90.3 & 87.9 & 0.46 \\
\hline
\end{tabular}

2 


\section{Table 6(on next page)}

Comparative performance between our classification method and BLAST search pipeline 
1 Table 6. Comparative performance between our classification method and BLAST search 2 pipeline

\begin{tabular}{|l|l|l|l|l|l|l|l|l|}
\hline \multirow{2}{*}{ Method } & \multicolumn{4}{|c|}{ Cross-validation } & \multicolumn{4}{c|}{ Independent } \\
\cline { 2 - 10 } & Sens & Spec & Acc & MCC & Sens & Spec & Acc & MCC \\
\hline BLAST & 37.0 & 99.3 & 85.3 & 0.53 & 26.3 & 99.4 & 92.2 & 0.44 \\
\hline 2D CNN & 76.6 & 93.5 & 89.7 & 0.7 & 65.8 & 90.3 & 87.9 & 0.46 \\
\hline
\end{tabular}

3 